\title{
Investigation of the effect of varying scatter-to- primary ratios on nodule contrast in chest tomosynthesis
}

\author{
Angelica Svalkvist, Gustaf Ullman, Markus Håkansson, David Dance, Michael Sandborg, \\ Gudrun Alm Carlsson and Magnus Båth
}

\section{Linköping University Post Print}

N.B.: When citing this work, cite the original article.

Original Publication:

Angelica Svalkvist, Gustaf Ullman, Markus Håkansson, David Dance, Michael Sandborg, Gudrun Alm Carlsson and Magnus Båth, Investigation of the effect of varying scatter-toprimary ratios on nodule contrast in chest tomosynthesis, 2011, Proc SPIE 7961, 79615Y.

http://dx.doi.org/10.1117/12.873173

Copyright 2011 Society of Photo-Optical Instrumentation Engineers. One print or electronic copy may be made for personal use only. Systematic reproduction and distribution, duplication of any material in this paper for a fee or for commercial purposes, or modification of the content of the paper are prohibited. 


\title{
Investigation of the effect of varying scatter-to-primary ratios on nodule contrast in chest tomosynthesis
}

\author{
Angelica Svalkvist*a ${ }^{\mathrm{a}}$, Gustaf Ullman ${ }^{\mathrm{b}, \mathrm{c}}$, Markus Håkansson ${ }^{\mathrm{a}, \mathrm{d}}$, David R. Dance ${ }^{\mathrm{e}}$, Michael Sandborg ${ }^{\mathrm{b}}$ \\ Gudrun Alm Carlsson ${ }^{\mathrm{b}}$, Magnus Båth ${ }^{\mathrm{a}, \mathrm{f}}$ \\ ${ }^{a}$ Dept. of Radiation Physics, Sahlgrenska Academy at University of Gothenburg, SE-413 45 \\ Gothenburg, Sweden \\ ${ }^{\mathrm{b}}$ Radiation Physics, Division of Radiological Sciences, Department of Medicine and Health \\ Sciences, Faculty of Health Sciences and Center for Medical Image Science and Visualization \\ (CMIV), Linköping University, SE-581 85 Linköping, Sweden \\ 'Department of Cell and Molecular Biology, Uppsala University, Uppsala, Sweden \\ ${ }^{\mathrm{d}}$ Dept. of Diagnostic Radiology, Södra Älvsborgs Sjukhus, Sahlgrenska University Hospital, SE-413 \\ 45 Gothenburg, Sweden \\ ${ }^{\mathrm{e}}$ NCCPM, Royal Surrey County Hospital, Guildford GU2 7XX, UK \\ ${ }^{\mathrm{f}}$ Dept. of Medical Physics and Biomedical Engineering, Sahlgrenska University Hospital, SE-413 45 \\ Gothenburg, Sweden
}

\begin{abstract}
The primary aim of the present work was to analyze the effects of varying scatter-to-primary ratios on the appearance of simulated nodules in chest tomosynthesis section images. Monte Carlo simulations of the chest tomosynthesis system GE Definium 8000 VolumeRAD (GE Healthcare, Chalfont St. Giles, UK) were used to investigate the variation of scatter-to-primary ratios between different angular projections. The simulations were based on a voxel phantom created from CT images of an anthropomorphic chest phantom. An artificial nodule was inserted at 80 different positions in the simulated phantom images, using five different approaches for the scatter-to-primary ratios in the insertion process. One approach included individual determination of the scatter-to primary-ratio for each projection image and nodule location, while the other four approaches were using mean value, median value and zero degree projection value of the scatter-toprimary ratios at each nodule position as well as using a constant scatter-to-primary ratio of 0.5 for all nodule positions. The results indicate that the scatter-to-primary ratios vary up to a factor of 10 between the different angular tomosynthesis projections $\left( \pm 15^{\circ}\right)$. However, the error in the resulting nodule contrast introduced by not taking all variations into account is in general smaller than $10 \%$.
\end{abstract}

Keywords: TSYN, XIM

\section{INTRODUCTION}

It has for long been known that overlapping anatomy is one of the main limiters for the possibility to detect pathology in radiological images. Despite this fact, projection radiography is still frequently used at the radiological departments. The use of computed tomography is one obvious way to efficiently reduce the problem of obscuring anatomy. However, due to the relatively high patient radiation dose, a CT examination can not always be justified. The introduction of tomosynthesis enables new possibilities in modern healthcare, as the technique reduces both the disadvantage of projection radiography (overlapping anatomy) and the disadvantage of CT (high radiation dose to the patients).

*angelica.svalkvist@vgregion.se; phone +4631 34240 27; fax +4631 34213 78; 
In tomosynthesis, a number of low-dose projection images are collected from different angles. These projection images are then used to create section images of the examined object, using modern reconstruction algorithms. In chest imaging, the tomosynthesis technique has, compared to conventional chest radiography, shown to be of great value in the detection of pulmonary nodules. ${ }^{1-3}$ However, no thorough description of the detection limitations of pulmonary nodules in chest tomosynthesis has yet been performed. Using only real, clinically found, nodules, it may be difficult to achieve the spectrum of nodule size, density and location necessary for a thorough evaluation. As previous studies ${ }^{4-7}$ have shown that hybrid images are a valuable complement to clinical images in detection studies, one solution could be to use simulated nodules in the evaluation. However, in order to motivate the use of hybrid images in evaluation of a clinical system it is important that the hybrid images are constructed in a way that reflects clinical reality. Therefore, it is important that the methods used to create the hybrid images are validated, i.e. it must be shown that no visual differences between real and simulated pathology can be found.

Our research group has previously described a method of simulating lung nodules in chest tomosynthesis ${ }^{8}$ and a small pilot study indicated that experienced radiologists had difficulties in separating the simulated nodules from real nodules. As a simplification, a scatter-to-primary ratio of 0.5 was assumed for the entire lung region. ${ }^{9}$ However, the scatter-toprimary ratio varies over the image and is higher close to the heart, diaphragm and central mediastinum. ${ }^{10-12}$ As the tomosynthesis projection images are collected from different angles, it can be anticipated that the scatter-to-primary ratio at the detector surface, for a given nodule, will vary between the different angular projections. Therefore it can be argued that the scatter-to-primary used when inserting the nodules into the tomosynthesis projection images not only should be adjusted according to the location in the parenchyma, but also according to the angle used for the different projection images.

The aims of the present work were to investigate the variation in scatter-to-primary ratio between different angular projections in chest tomosynthesis and to analyze the effects of varying scatter-to-primary ratios on the appearance of simulated nodules in the tomosynthesis section images.

\section{MATERIAL AND METHODS}

\subsection{Tomosynthesis system description}

At our hospital the Definium 8000 X-ray system with VolumeRAD option (GE Healthcare, Chalfont St. Giles, UK) is used for chest tomosynthesis examinations. During a tomosynthesis examination using this system, the detector position is fixed, whereas the x-ray tube performs a vertical continuous movement from $-17.5^{\circ}$ to $+17.5^{\circ}$ relative to the standard orthogonal posteroanterior (PA) projection. During a time period of approximately ten seconds, the system collects 60 low-dose projection images of the patient in the angular interval of $-15^{\circ}$ to $+15^{\circ}$. The focus-detector distance is $180 \mathrm{~cm}$ in the PA projection. For all projection images a tube voltage of $120 \mathrm{kV}$ and a filtration of $3 \mathrm{~mm} \mathrm{Al}+0.1 \mathrm{~mm} \mathrm{Cu}$ are used. For the GE VolumeRAD system, the exposure used during a tomosynthesis examination is determined by an initial scout image of the patient. The scout image corresponds to a normal PA chest radiograph and is collected using automatic exposure control. The exposure used for the scout image is then multiplied with a factor (normally ten) and evenly distributed over the 60 projection images. The exposure is then rounded down to the closest mAs setting possible, using the constraint of a minimum tube load of $0.25 \mathrm{mAs}$ per projection. The detector size is $2022 \times 2022$ pixels, with a pixel size of $0.2 \times 0.2 \mathrm{~mm}^{2}$, and the focal spot size is $1.25 \times 1.25 \mathrm{~mm}^{2}$.

\subsection{Nodule simulation}

A method for simulation of lung nodules in chest tomosynthesis has previously been described by Svalkvist et al. ${ }^{8}$ The method is based on the principle of creating 3-dimensional artificial nodules, which are inserted into the 60 projection images before reconstruction of the tomosynthesis section images. In order to obtain a realistic appearance of the simulated nodules, both an irregular shape and surface structure of the simulated nodules are desirable. This is achieved by using an approach of combining spheres of different sizes. Thus, the nodule is created by starting with an original sphere and then adding a number of additional, smaller spheres to the original sphere in two steps: first, to create the nodule shape and second, to create the surface structure. Randomization of the number, size and location of the additional spheres that are added to the original sphere results in a unique appearance for each created nodule. After each addition of spheres to the original sphere the result is smoothed using a mean filter to obtain a softer and more realistic 
appearance of the simulated nodule, see Figure 1. The volume of each created nodule is determined by the volume of the original sphere. Thus, after adding additional spheres to the original sphere, the resulting nodule is scaled down to a volume corresponding to the volume of the original sphere.

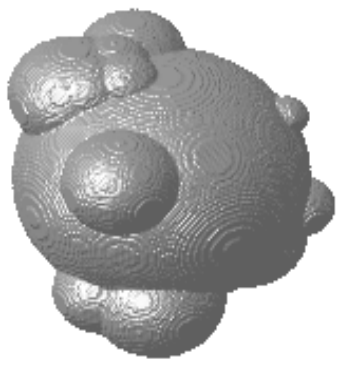

a

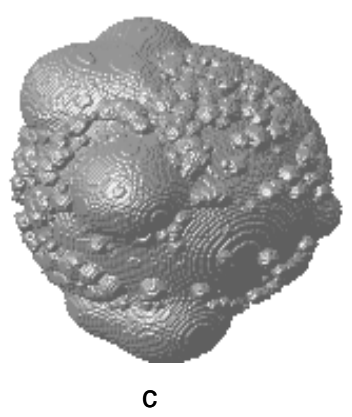

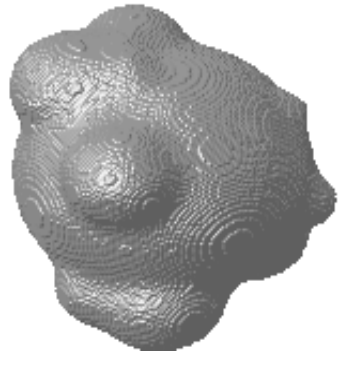

b

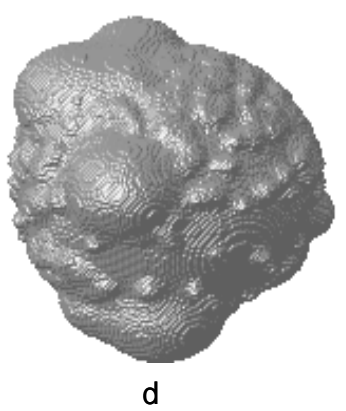

Figure 1. Example of the step-by-step creation of a simulated nodule. (a) Additional spheres added to an original sphere in order to create the shape of the nodule. (b) After smoothing of the created shape. (c) Additional spheres added to the created nodule shape in order to create the surface structure of the nodule. (d) The final resulting nodule after smoothing.

The simulated nodules are inserted into the raw-data projection images of a tomosynthesis acquisition before reconstruction of the tomosynthesis section images. Svalkvist et al. ${ }^{8}$ describe how the desired position of the simulated nodule in the patient and the geometry of the tomosynthesis acquisition are used to calculate the corresponding position of the nodule in each raw-data projection image. The insertion of the nodule into each projection image is then performed by simulating the radiation that is emitted from the focal spot, passes through the nodule and reaches the detector.

In order to obtain the correct contrast level of the simulated nodules in the tomosynthesis projection images, the signal spread in the detector, the scattered radiation and the patient motion must be accounted for. As described in Svalkvist et $\mathrm{al}^{8}$ the amount of patient motion varies both between patients and between different areas of the lung. The amount of patient motion present at a specific location in a patient is difficult to quantify. However, by analyzing the borderline of e.g. blood vessels in the close proximity of the nodule location, the amount of patient motion can be estimated. In Svalkvist et al., the patient motion is accounted for by randomly shifting the location of the nodule before projecting the nodule into each projection image. Consequently, the more patient motion present at the location where the nodule is to be inserted, the larger the shifting of the nodule centre between each projection image.

The amount of signal spread in the detector can be accounted for by applying the two-dimensional pre-sampling modulation transfer function (MTF) of the detector to the signal before sampling. The contrast degradation due to scattered radiation can be accounted for by adjusting the signal according to the known scatter-to-primary ratio. 


\subsection{Creation of the Monte Carlo simulated tomosynthesis projection images}

As described above, in addition to the signal spread in the detector and the patient motion, the signal contribution from scattered radiation will affect the appearance of the simulated nodules in the final reconstructed tomosynthesis section images. One way to analyze the variation in the scatter-to-primary ratio, both between different angular projections and between different regions of the lung parenchyma, is to simulate the imaging chain using the Monte Carlo technique. In this work, Monte Carlo simulations of the GE Definium VolumeRAD tomosynthesis system, described by Ullman et al. ${ }^{13}$, were used. In the work by Ullman et al., all simulations were based on a voxel phantom, which was created by scanning an anthropomorphic chest phantom (Kyoto Kagaku PBU-X-21) in a Siemens Sensation 64 CT scanner at a tube voltage of $120 \mathrm{kV}$. The images were reconstructed using the kernel B31s and segmented using a step density function with a set of thresholds for Hounsfield values corresponding to lung, soft and bone tissues. The simulation geometry and input parameters were adjusted to match the VolumeRAD chest tomosynthesis system. The projection images due to primary photons were calculated analytically with the resolution $2022 \times 2022$ pixels, the same as in the VolumeRAD system. This was done using an analytical model of the anti-scatter grid and a pre-calculated detector response function. ${ }^{14}$ The Monte Carlo model used for estimation of the scatter-to-primary ratios simulates photons that starts from a point source and subsequently passes through both the anthropomorphic voxel phantom and the anti-scatter grid before finally reaching the image detector. In order to save computational time, the signal contributions from scattered photons were calculated in a coarser grid of $40 \times 40$ points, for each tomosynthesis projection angle. The scatter projections were then added to the projection images in order to obtain simulated tomosynthesis projection images with realistic clinical properties. In Figure 1 (a-c) the variation in scatter-to-primary ratio for three of the 60 tomosynthesis projection images (projection angles $-15^{\circ}, 0^{\circ}$ and $+15^{\circ}$ respectively) resulting from the Monte Carlo simulations are shown.
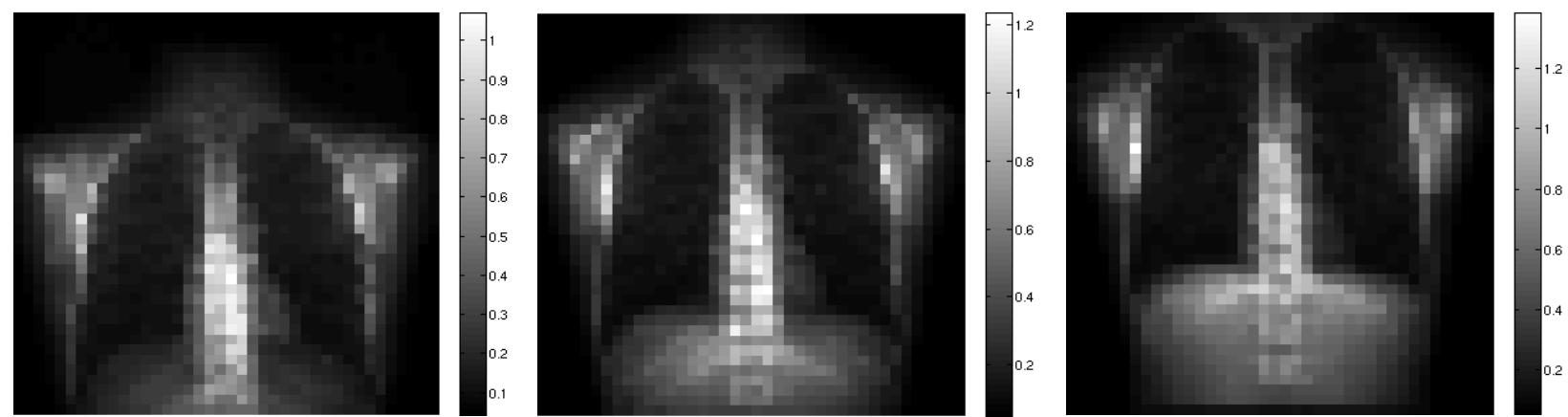

Figure 2. The variation in scatter-to-primary ratio for three of the 60 tomosynthesis projection images resulting from the Monte Carlo simulations (left $-15^{\circ}$, middle $0^{\circ}$ and right $+15^{\circ}$ ).

\subsection{Analysis of the scatter-to-primary ratio used for the nodule insertion}

In order to match the resolution of the tomosynthesis projection images, the scatter-to-primary ratios from Ullman et al. ${ }^{13}$ were interpolated to $2022 \times 2022$ points using bilinear interpolation. By using the method described by Svalkvist et al. ${ }^{8}$, an artificial nodule of $12 \mathrm{~mm}$ in diameter was created. The nodule was then inserted at 80 positions of the Monte Carlo simulated tomosynthesis projection images. The positions were chosen to represent 20 locations in four different depths of the phantom. Different approaches regarding the scatter-to-primary ratio used when inserting the artificial nodule into each projection image were investigated: 1 . the scatter-to-primary ratio was determined individually for every projection image and nodule position, 2. the mean value of the scatter-to-primary ratio for all projection images at each position was used, 3. the median scatter-to-primary ratio for all projection images at each position was used, 4. the scatter-toprimary ratios for the $0^{\circ}$-projection at each nodule position was used, and 5 . a constant scatter-to-primary ratio of 0.5 was used for all projection images and nodule positions. ${ }^{9}$ After the insertion of the artificial nodule, the Monte Carlo simulated projection images were transferred to the VolumeRAD system for reconstruction.

As the reconstructed tomosynthesis section images could not be confirmed being linear, a scaled contrast, C, of the inserted nodule was determined as: 


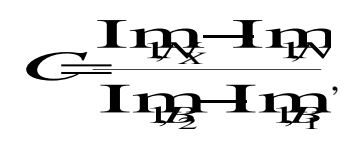

where $\operatorname{Im}_{1, \mathrm{Nx}}$ is the mean pixel value in an image 1 at the position of an inserted nodule and $\operatorname{Im}_{1, \mathrm{~N}}$ is the mean pixel value at the same position, had the nodule not been inserted. $\operatorname{Im}_{1, \mathrm{~B} 1}$ and $\operatorname{Im}_{1, \mathrm{~B} 2}$ are the mean pixel values at two arbitrarily chosen positions in the background. The scaled contrast thus relates the differential signal from the nodule to a given background difference. $\operatorname{Im}_{1, \mathrm{~N}}$ cannot be measured in the image. However, if the background positions are chosen so that the numerator and denumerator become similar in size and all terms on the right hand side of Eq. (1) are of similar value, a small signal approximation enables the following relationship - where $\operatorname{Im}_{0}$ is an image reconstructed without the inserted simulated nodule - to be valid even if the reconstructed section images cannot be assumed to be linear:

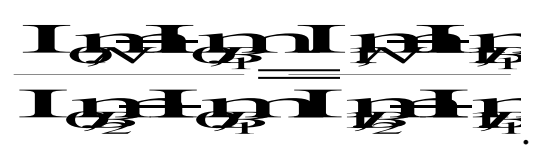

Hence, $\operatorname{Im}_{1, \mathrm{~N}}$ can be determined by:

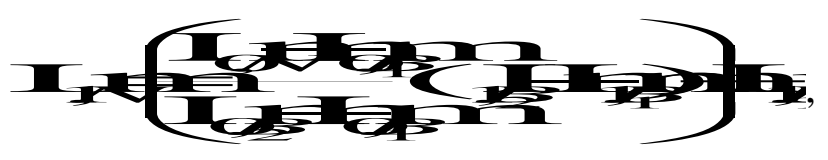

where all the values on the right hand side of the equation can be measured. The mean pixel value at the different positions in the reconstructed images were determined using a region of interest (ROI) of diameter $8 \mathrm{~mm}$.

\section{RESULTS}

Figure 3 shows one of the tomosynthesis section images with a $12 \mathrm{~mm}$ artificial nodule inserted at multiple locations in the phantom and the mean, median and range of the scatter-to-primary ratio for all 60 projection images at each nodule location. The results indicate that the scatter-to-primary ratio varies both between the different projection images and between different regions of the lung. In locations close to density borders, e.g. close to the diaphragm and heart, a wider range of variation in scatter-to-primary ratios between the 60 projection images could be seen. In certain regions, e.g. nodule locations nos. 10 and 11, the range of scatter-to-primary ratios covered a factor of 10 . In these regions also the difference between the mean scatter-to-primary ratio and median scatter-to-primary ratio was larger. 


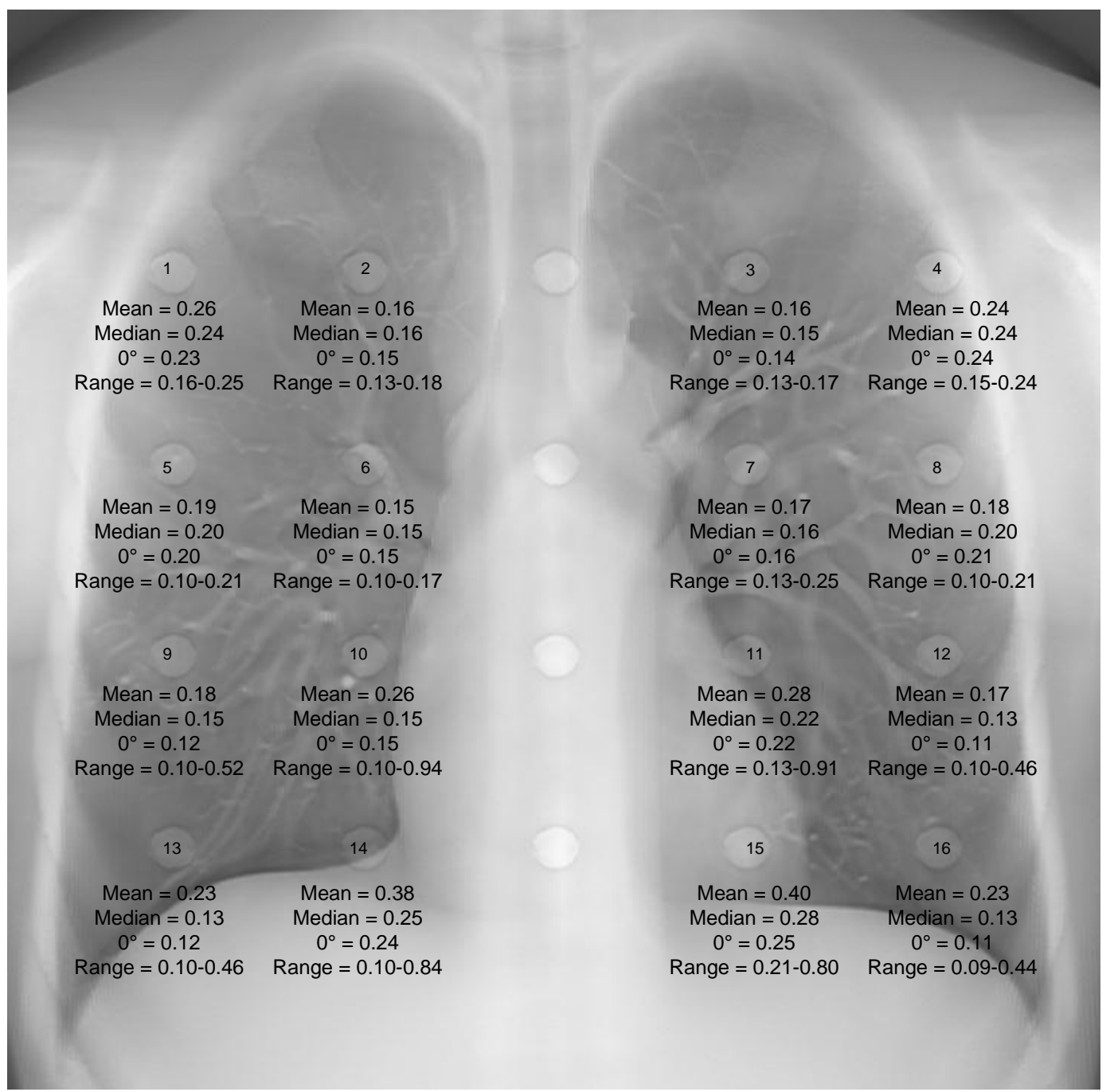

Figure 3. A reconstructed tomosynthesis section image with a $12 \mathrm{~mm}$ artificial nodule inserted at 20 different positions in the phantom. The mean value, median value, $0^{\circ}$-projection value and range of the scatter-to-primary ratios for the individual projection images $\left( \pm 15^{\circ}\right)$ are shown.

In Figure 4 the effects on nodule contrast of using the different approaches for the scatter-to-primary ratios in the simulations, are shown for each of the nodule locations presented in Figure 3. The error in scaled nodule contrast when using the mean value, median value or the $0^{\circ}$-projection value of the scatter-to-primary ratios in the nodule simulations, instead of values individually adjusted for each projection image and nodule location, was in general smaller than $10 \%$. Exceptions were found for nodules located close to density borders, for example nodule locations 4 and 14 . At most nodule locations, using a constant scatter-to-primary value of 0.5 in the nodule simulations resulted in a nodule with a lower contrast, compared to adjusting the scatter-to-primary ratio individually for each projection image and nodule location. 


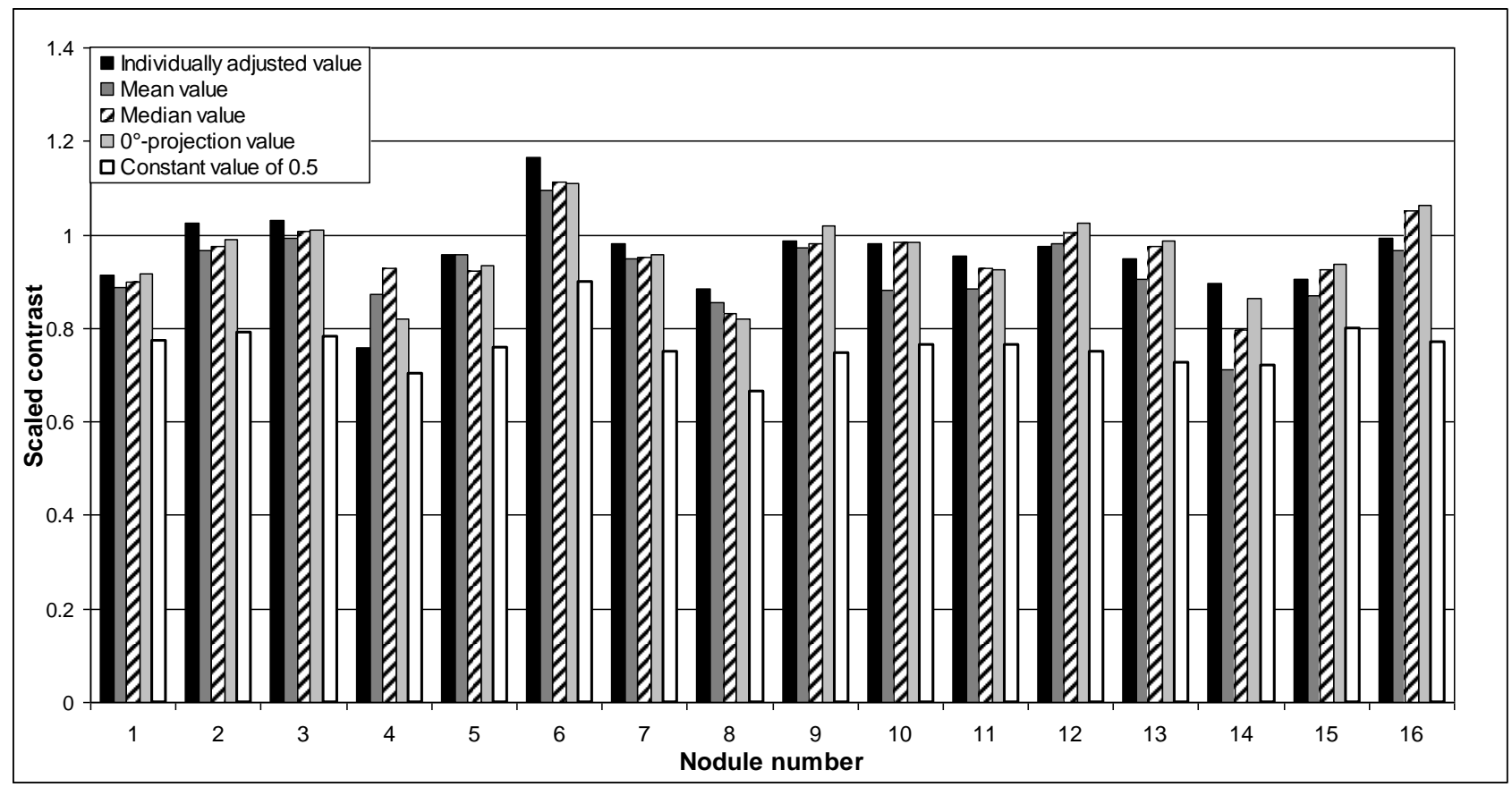

Figure 4. Variation in scaled contrast of the nodule locations presented in Figure 1, using the different approaches for determining the scatter-to-primary ratio in the nodule simulation process.

In Table 1, the scaled nodule contrasts obtained using individually adjusted scatter-to-primary ratios are presented for each nodule location, at various depths of the phantom. In addition, Table 1 presents the relative error in scaled nodule contrast obtained using the other approaches regarding scatter-to-primary ratio (2-5 above) in the nodule simulation process. The relative error was larger for nodules located close to density borders and a variation was found between different depths of the phantom. Note that no comparison can be made between the actual contrast values at the different depths of the phantom, as the scaling of the nodule contrast is unique for each tomosynthesis section image. 
Table 1. The scaled nodule contrasts obtained at each nodule location, at various depths in the phantom, when using individually adjusted scatter-to-primary ratios in the nodule simulation, and the relative error in using the other approaches. Numbers marked in grey correspond to nodule locations outside of
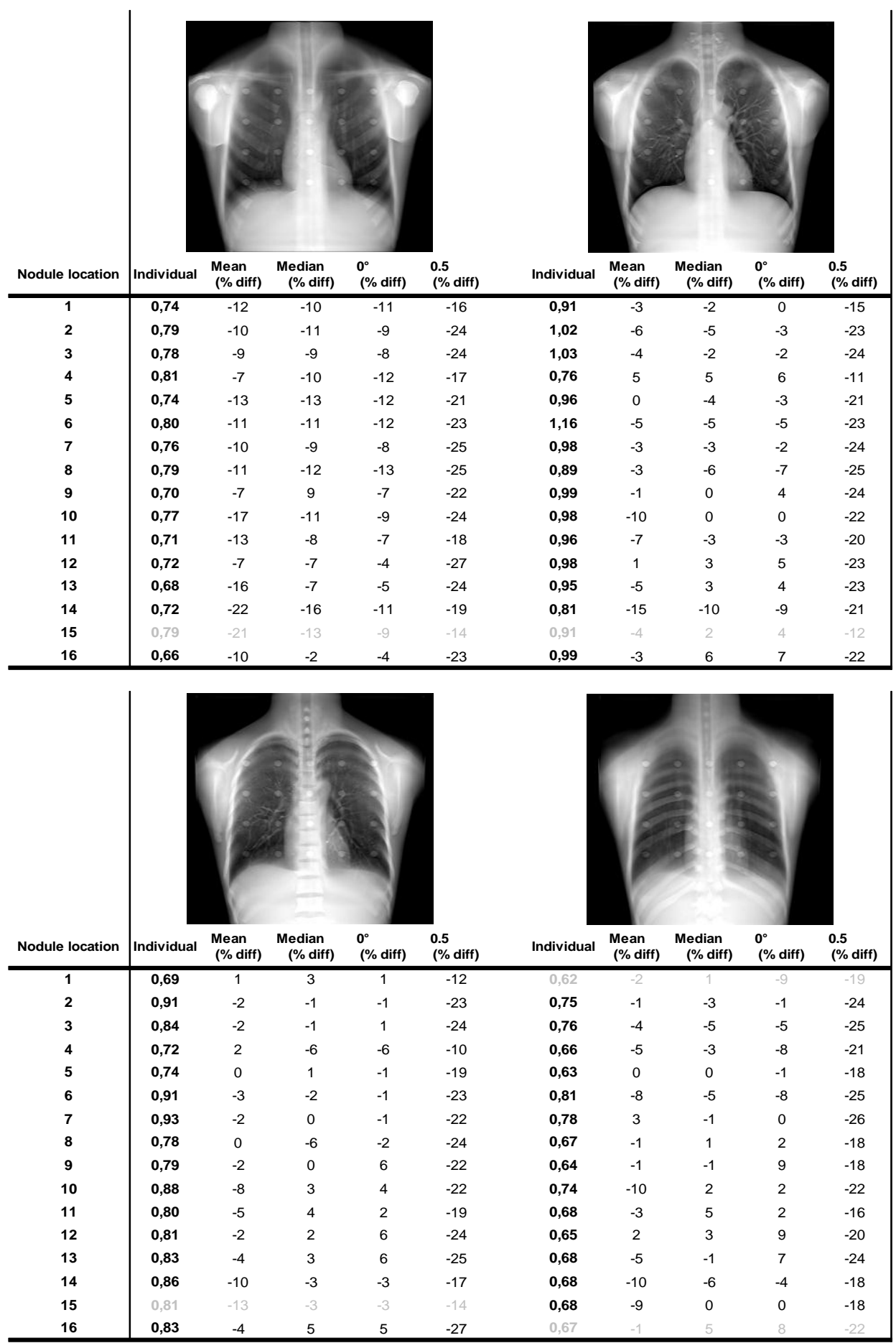

the lung parenchyma. Note: Scaled contrast values cannot be compared between depths. 


\section{DISCUSSION}

The first aim of the present paper was to investigate the variation in scatter-to-primary ratio between different angular projections in chest tomosynthesis. By using Monte Carlo simulations of an anthropomorphic chest phantom, the signal contribution from scattered photons in each projection image included in a chest tomosynthesis examination could be estimated and compared. The second aim of this work was to analyze the effect of varying scatter-to-primary ratios on the appearance of simulated structures in the tomosynthesis section images. A method for simulating lung nodules in chest tomosynthesis was used to investigate the effect on nodule contrast when using different approaches for the scatterto-primary ratio in the simulation process.

The evaluation of the variation of scatter-to-primary ratio between the projection images revealed a larger variation in scatter-to-primary ratio between the projection images in locations close to density borders. The range of the scatter-toprimary ratio was especially large close to the diaphragm and heart; see e.g. locations 10, 11, and 14 in Figure 3. However, as seen in Figures 3 and 4, the variation in contrast obtained for a simulated nodule, using the five different approaches regarding the scatter-to-primary ratio in the simulation process, was smaller. The relative error obtained using the mean value, median value or the $0^{\circ}$-projection value of the scatter-to-primary ratio, instead of adjusting the scatter-to-primary ratio individually for each projection image and nodule location, was in general smaller than $10 \%$. Using a constant scatter-to-primary value of 0.5 resulted in a nodule with too low a contrast, although the error (approx. 20\%) was relatively constant, indicating the possibility of using a constant scatter-to-primary ratio in the entire parenchyma and only adjusting the value to the size of the patient. It should be noted that no quantum noise were added to the Monte Carlo simulated projection images used in the present work and the uncertainties related to the Monte Carlo simulations were approximately $2 \%$ for each projection image (one standard deviation).

In the present paper, the nodule locations in the phantom were chosen to cover most areas of the lung parenchyma. As the results indicate that the error in scaled contrast, when using the mean value, median value or $0^{\circ}$-projection value in the nodule simulation, is larger for nodules located close to density borders, it can be anticipated that the relative errors presented in the present paper may not be representative for the entire lung parenchyma. It must also be noted that the voxel phantom used for Monte Carlo simulations of the tomosynthesis system is relatively small compared to a medium sized patient. Hence, the scatter-to-signal ratios presented in the present work may be slightly underestimated. Also, it cannot be certified that the variation in scatter-to-primary ratio will be the same in a larger patient.

\section{CONCLUSIONS}

The scatter-to-primary ratios at the detector surface, for a specific location in the patient, will vary up to a factor of 10 between the different angular tomosynthesis projections $\left( \pm 15^{\circ}\right)$. However, the error in the resulting nodule contrast introduced by not taking all variations into account is much smaller. For example, using the scatter-to-primary ratio for the $0^{\circ}$-projection for all projection images results in an error in nodule contrast that in general is smaller than $10 \%$. The use of a constant scatter-to-primary ratio of 0.5 in the entire lung parenchyma resulted in a relatively constant error, indicating the possibility of only adjusting the scatter-to-primary ratio for the size of the patient. 


\section{REFERENCES}

[1] Dobbins J.T., McAdams, H.P., Song, J.W., Li C.M., Godfrey D.J., DeLong, D.M., Paik, S.-H. and MartinezJimenes, S., "Digital tomosynthesis of the chest for lung nodule detection: Interim sensitivity results from an ongoing NIH-sponsored trial," Med. Phys. 35(6), 2554-2557 (2008).

[2] Vikgren, J., Zachrisson, S., Svalkvist, A., Johnsson, Å.A., Boijsen, M., Flinck, A., Kheddache, S. and Båth, M., "Comparison of chest tomosynthesis and chest radiography for detection of pulmonary nodules: Human observer study of clinical cases," Radiology 249(3), 1034-1041 (2008).

[3] Quaia, E., Baratella, E., Cioffi, V., Bregant, P., Cernic, S., Cuttin, R. and Cova, M.A., "The value of digital tomosynthesis in the diagnostics of suspected pulmonary lesions on chest radiography: Analysis of diagnostic accuracy and confidence," Acad. Radiol. 17(10), 1267-1274 (2010).

[4] Samei, E., Flynn, M.J. and Eyler, W.R., "Detection of subtle lung nodules: relative influence of quantum and anatomic noise on chest radiographs," Radiology 213(3), 727-734 (1999).

[5] Burgess, A.E., Jacobson, F.L. and Judy, P.F., "Human observer detection experiments with mammograms and power-law noise," Med. Phys. 28(4), 419-437 (2001).

[6] Båth, M., Håkansson, M., Börjesson, S., Kheddache, S., Grahn, A., Ruschin, M., Tingberg, A., Mattsson, S. and Månsson, L.G., "Nodule detection in chest radiography: Introduction to the RADIUS chest trial," Radiat. Prot. Dosimetry 114, 85-91 (2005).

[7] Håkansson, M., Båth, M., Börjesson, S., Kheddache, S., Grahn, A., Ruschin, M., Tingberg, A., Mattsson, S. and Månsson, L.G., "Nodule detection in chest radiography: Summaary of the RADIUS chest trial," Radiat. Prot. Dosimetry 114, 114-120 (2005).

[8] Svalkvist, A., Håkansson, M., Ullman, G. and Båth, M., "Simulation of lung nodules in chest tomosynthesis," Radiat. Prot. Dosimetry 139, 130-139 (2010).

[9] Dobbins III, J.T., [Handbook of Medical Imaging], SPIE Press, Bellingham, Washington, 161-222 (2000).

[10] Ullman, G., Sandborg, M., Dance, D.R., Hunt, R. and Alm-Carlsson, G., "Distributions of scatter-to-primary and signal-to-noise ratios per pixel in digital chest imaging,", Radiat. Prot. Dosimetry 114, 355-358 (2005).

[11] Niklason, L.T., Sorenson, J.A., and Nelson, J.A., "Scattered radiation in chest radiography," Med. Phys. 8(5), 677-681 (1981).

[12] Jordan III, L.K., Floyd Jr., C.E., Lo, J.Y. and Ravin, C.E., "Measurement of scatter fractions in erect posteroanterior and lateral chest radiography," Radiology 188, 215-218 (1993).

[13] Ullman, G., Dance, D. R., Sandborg, M., Carlsson, G. A., Svalkvist, A. and Båth, M., "A Monte Carlo-based model for simulation of digital chest tomosynthesis," Radiat. Prot. Dosimetry 139, 159-163 (2010).

[14] Sandborg, M., Dance, D.R., Persliden, J. and Carlsson, G.A., "A Monte Carlo program for the calculation of contrast, noise and absorbed dose in diagnostic radiology," Comput. Methods Programs Biomed. 42(3), 167180 (1994). 